\title{
On Minimum-Collisions Assignment in Heterogeneous Self-Organizing Networks
}

\author{
Mathew Goonewardena *, Hoda Akbari ${ }^{\S}$, Wessam Ajib ${ }^{\dagger}$, and Halima Elbiaze ${ }^{\dagger}$ \\ ${ }^{* \dagger}$ Department of Computer Science, Université du Québec à Montréal (UQAM), Quebec, Canada, \\ $\S$ School of Computing Science, Simon Fraser University (SFU), Burnaby, British Columbia, Canada, \\ * mathew-pradeep.goonewardena.1@ens.etsmtl.ca, ${ }^{\S}$ hodaa@ sfu.ca, ${ }^{\dagger}\{$ ajib.wessam, elbiaze.halima $\} @$ uqam.ca
}

\begin{abstract}
Minimum-collisions assignment, in a wireless network, is the distribution of a finite resource set, such that the number of neighbor cells which receive common elements is minimized. In classical operator deployed networks, resources are assigned centrally. Heterogeneous networks contain user deployed cells, therefore centralized assignment is problematic. This paper examines the minimum-collisions assignment problem in the context of physical cell identity (PCI) allocation. Minimumcollisions assignment is NP-complete, therefore a potential-gametheoretic model is proposed as a distributed solution. The players of the game are the cells, actions are the set of PCIs and the utility of a cell is the number of neighbor cells in collision. The price of anarchy and price of stability are derived. Moreover the paper adapts a randomized-distributed-synchronous-update algorithm, for the case, when the number of PCIs is higher than the maximum degree of the neighbor relations graph. It is proven that the algorithm converges to a optimal pure strategy Nash equilibrium in finite time and it is robust to node addition. Simulation results demonstrate that the algorithm is sub-linear in the size of the input graph, thus outperforms best response dynamics.
\end{abstract}

\section{INTRODUCTION}

In a heterogeneous and small cell wireless network (HetSNet), user deployed small cells (SCs), also known as femtocells, must coexist with the operator managed larger cells. Just as any cell, SCs too require operating parameters in order to integrate to the wireless network. The sheer numbers of SCs and the unpredictability of their on-off times have increased the research interest in self-organizing networks (SONs) [1]. In a SON the network intelligence is distributed among the cells and interactions among the cells define the state to which the network stabilizes. This paper analyzes the equilibrium performance of a self-organizing HetSNet, for the problem of minimum-collisions assignments.

Consider an undirected graph $G:=(\mathcal{M}, \mathcal{E})$, where $\mathcal{M}$ is the set of vertices and $\mathcal{E}$ is the set edges. Minimum-collisions assignments is the problem, where the objective is to allocate a finite set of resources $\mathcal{N}$ over the vertices of the graph $G$, such that the sum collisions are minimized. A collision is a situation where two vertices, which share an edge, obtain a common element from $\mathcal{N}$. Examples of minimum-collisions assignment problems are, physical cell identity (PCI) assignment, orthogonal frequency band distribution among cells and scheduling time slots of a finite duration frame over a set of collocated links. The rest of the paper refers to the resource set $\mathcal{N}$, as the set of PCIs and minimum-collisions assignment is synonymously called PCI assignment. PCIs are a limited set of addresses, which is generally lower in number than the cells in the network [2].

Minimum-collisions assignment is NP-complete. Consider a reduction from classical graph coloring problem; given a graph $G=(\mathcal{M}, \mathcal{E})$ and a positive integer $k$, can the graph be $k$-colored? Let this problem be $P_{1}(G, k)$. On the other hand the decision problem of minimum-collisions assignment can be formulated as follows; given the graph $G$ and two positive integers $k$ and $\tau$, is there an assignment of $k$ colors to the nodes such that the number of collisions is at most $\tau$ ? This problem is denoted by $P_{2}(G, k, \tau)$. First it is clear that $P_{2}$ is NP. Then note that $P_{1}(G, k)$ is reduced, in polynomial time, to $P_{2}(G, k, 0)$, as $P_{1}$ is NP-complete, it follows that $P_{2}$ is NPhard. In conclusion since $P_{2}$ is NP-hard and is in NP, it is NPcomplete. NP-completeness of the problem justifies seeking heuristic solutions. This paper is concerned with scalable, distributed, heuristic based on the theory of games. Nash equilibrium (NE) is a widely accepted stable solution that characterizes the end result of the interactions among rational agents. The NE PCI assignments provide the network designer a game-theoretical justification of the expected functionality of the network if the control is decentralized among the players.

While minimum-collisions assignment resembles a variation of graph coloring, the objective of the two optimization problems are considerably different. In classical graph coloring optimization, the objective is to minimize the number of colors used, always having sufficient colors to achieve zero collisions [3]. On the other hand, in minimum-collisions assignment, the number of colors (PCIs) is constant and the objective is to minimize the collisions. There is no motivation to minimize the number of of PCIs used and equally there is no space to scale up the number of PCIs. This key difference sets our research apart from the previous game-theoretic research on coloring problem and for these reasons, the price of anarchy bound of [3] is not applicable to the objective of this paper.

Yet studying graph coloring problem is useful for some cases of PCI assignment, where a zero collision is known to exist, especially $|\mathcal{N}|>\Delta(G)$, where $\Delta(G)$ is the maximum degree of the graph $G$. In [3], the classical graph coloring problem is formulated as a potential game played by the vertices. The case considered is when the number of nodes are equal to the number of colors, i.e., $|\mathcal{M}|=|\mathcal{N}|$. The considered social cost is the number of colors used by all the players. Clearly in PCI assignment the interest lies in the case when 
$|\mathcal{M}| \gg|\mathcal{N}|$ and hence [3] is not directly applicable. Improved bounds for the worst case number of colors required at a NE, under the same setup as in [3], is presented in [4].

A direct adaptation of the algorithm of [5] for the signal to interference plus noise ration (SINR) model is analyzed in [6]. In [7], the problem of assigning a fixed number of channels among base stations (BSs), so that adjacent BSs do not receive the same channel is considered as a game. In [8], a potential game is introduced for frequency assignment. Their model is based on SINR and the utility is a function of interference. The game discussed in [9], has been modeled to maximize the use of a resource, whereas in our paper the objective is to minimize the use of the same resource among neighbors. There are a number of research works which propose heuristic algorithms for PCI assignment. The algorithm proposed in [10], is decentralized and depends on BSs exchanging neighbor relation tables as opposed to the algorithm in [11], which is centralized and makes use of messages from UEs to build the neighbor relation tables and PCI assignment. In [12], a dynamic PCI assignment algorithm is proposed. The algorithm categorizes the BSs according to activity level and assigns a unique PCI to busy cells while cells with lower activity obtains a reused PCI. PCI assignment as a graph coloring problem is considered in [13], [14]. To the best of our knowledge this is the first paper to present a potentialgame theoretic analysis for PCI assignment.

In addition this paper proposes a randomized distributed algorithm to achieve optimal NE, when $|\mathcal{N}|$ is at least $\Delta(G)+$ 1 , and proves it to converge in finite time. The algorithm is an improvement upon [15], where the proposed algorithm is shown unable to achieve NE for $|\mathcal{N}|=\Delta(G)+1$. Algorithm of [16] too requires $\Delta(G)+2$ colors for some instances and both, [15], [16] require memory of colors used by neighbors. The proposed algorithm is robust, that is nodes, which have already acquired a color need not change if a downed node reappears in the graph $G$. Compared to [17], the algorithm of this paper requires communication only among neighbors.

The remainder of the paper is organized as follows. Section II develops the non-cooperative potential game for the problem. Section III analysis the game for price of stability and price of anarchy. Section IV proposes the algorithm and proves convergence. Section $\mathrm{V}$ presents numerical results of simulations and finally Section VI concludes the paper.

\section{PCI ASSIGNMENT GAME}

This paper considers a HetSNet which consists of a set $\mathcal{M}:=\{1, \ldots, M\}$ of BSs. To avoid trivial instances $M \geq 2$. The PCI resource set is $\mathcal{N}:=\{1, \ldots, N\}$. The PCI of BS $m$ is denoted by $s_{m}$. The generation of the graph $G=(\mathcal{M}, \mathcal{E})$, has to reflect the requirements of the problem. In the particular case of PCI assignment, the set of edges $\mathcal{E}$, must contain the cells with overlapping coverage areas, to denote possible conflicts. In addition, the edge set also must contain the possible PCI confusions by introducing an edge between two cells which have a common neighbor, even if these two cells may not have common coverage area [18]. As shown in the example given in Fig. 1a, edge $(d, f)$ has to be in $G$, to denote

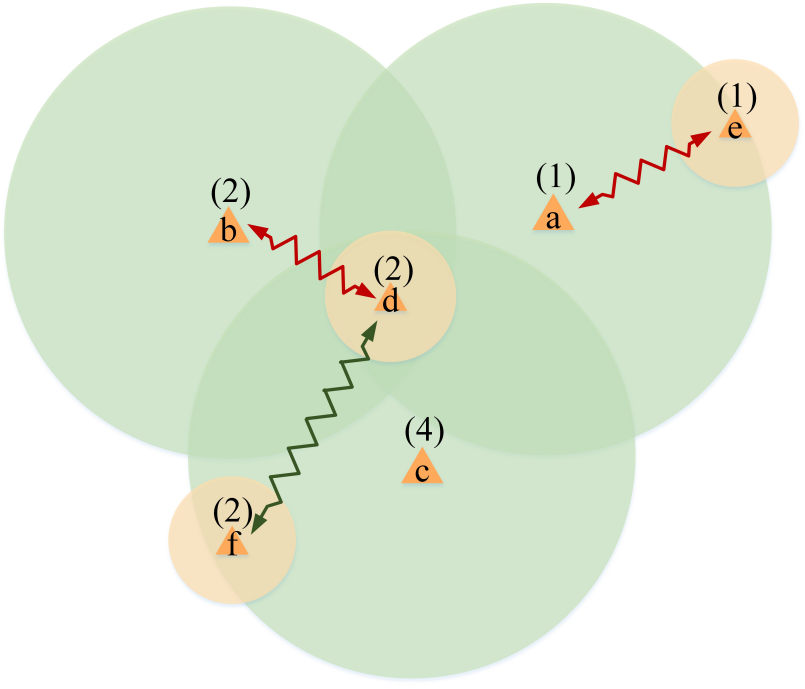

(a) For the given PCI assignment, the red and green wiggly arrows denote conflicts and confusions respectively. PCI number is shown above the BS in parenthesis.

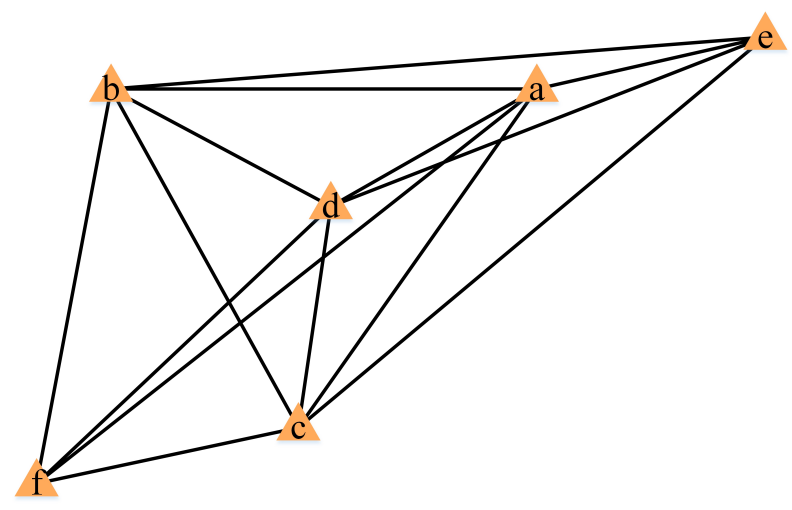

(b) The only edge not included in neighbor relations graph $G$ is $(d, f)$. Having the same PCI at nodes $d$ and $f$ does not create collisions.

Figure 1: A HetSNet and the corresponding neighbor relations graph $G$.

that if BSs $d$ and $f$ obtain a common value, then BS $c$ has a confusion. In this paper, both conflicts and confusions are commonly identified as collisions. Therefore to build the edge set of the graph $G$, one can simply assign a single PCI to all BSs $\mathcal{M}$, and create an edge between each pair of BSs, which are in collision. Fig. $1 \mathrm{~b}$ represents the equivalent neighbor relations graph $G$, for the network of Fig. 1a. Let $\mathcal{M}_{m}$ denote the set of neighbors of BS $m \in \mathcal{M}$, i.e., all BSs which have an edge with $m$, and let cardinality $\left|\mathcal{M}_{m}\right|=M_{m}$. Suppose there is no central assignment and instead each BS selfishly chooses. This can be modeled by a non-cooperative game where the players are the BSs. This paper considers an affine cost function in the number of collisions. The cost of BS $m \in \mathcal{M}$ for choosing PCI $s_{m} \in \mathcal{N}$ is

$$
c_{m}\left(s_{m}, \mathbf{s}_{-m}\right):=a\left|\left\{m^{\prime}: s_{m^{\prime}}=s_{m}, m^{\prime} \in \mathcal{M}_{m}\right\}\right|+d,
$$

where $a$, and $d$ are positive valued constants, the set $\left\{m^{\prime}: s_{m^{\prime}}=s_{m}, m^{\prime} \in \mathcal{M}_{m}\right\}$ is the neighbors of $m$ with the same PCI as $s_{m}$. 
Following the standard notation of game theory, the vector $\mathbf{S}_{-m}$ denotes the choices of all other BSs except $m$. Let $\mathbf{s}:=$ $\left(s_{m}, \mathbf{s}_{-m}\right)$. Then the total cost, also called the social cost, of the system is the sum cost of the set of BSs,

$$
C(\mathbf{s}):=\sum_{m \in \mathcal{M}} c_{m}\left(s_{m}, \mathbf{s}_{-m}\right) .
$$

The finite strategy normal form game, which is performed by the BSs, is defined by $\mathcal{G}_{\mathcal{M}}:=$ $\left\langle\mathcal{M}, \mathcal{N}^{M},\left\{c_{m}(\cdot) \mid m \in \mathcal{M}\right\}\right\rangle$, where $\mathcal{N}^{M}$ is the action space of the $M$ players.

To prove the existence of pure strategy NEs of $\mathcal{G}_{\mathcal{M}}$, this paper relies on the subclass of non-cooperative games called potential games. Potential games are identified by having a potential function [19].

Definition 1. (Weighted potential function) A function $\phi: \mathcal{N}^{M} \rightarrow \mathbb{R}$, is a weighted potential function of $\mathcal{G}_{\mathcal{M}}$, with weight $w$, if $\forall m \in \mathcal{M}, \forall n, n^{\prime} \in \mathcal{N}$,

$\phi\left(n, \mathbf{s}_{-m}\right)-\phi\left(n^{\prime}, \mathbf{s}_{-m}\right)=w\left(c_{m}\left(n, \mathbf{s}_{-m}\right)-c_{m}\left(n^{\prime}, \mathbf{s}_{-m}\right)\right)$.

It is well known that all finite games with a potential function posses at least one pure strategy NE and the pure strategy NEs are the local minima of the potential function [19]. Furthermore in a finite game with a potential function, the sequential best response (BR) dynamics always converge to a NE [19]. The following lemma claims that $\mathcal{G}_{\mathcal{M}}$ has a weighted potential function and there for posses a NE.

Lemma 1. $\mathcal{G}_{\mathcal{M}}$ has a pure strategy $N E$.

Proof: The change in cost of player $m$ as it changes its choice, from $s_{m}=n$ to $s_{m}=n^{\prime}, n, n^{\prime} \in \mathcal{N}$, while all other players hold their PCIs, (i.e., $\mathbf{s}_{-m}$ constant) is,

$$
\begin{aligned}
\Delta c_{m}\left(\hat{\curvearrowright n^{\prime}}, \mathbf{s}_{-m}\right) & =c_{m}\left(n^{\prime}, \mathbf{s}_{-m}\right)-c_{m}\left(n, \mathbf{s}_{-m}\right) . \\
\Delta c_{m}\left(\hat{n n^{\prime}}, \mathbf{s}_{-m}\right)= & a\left|\left\{m^{\prime}: s_{m^{\prime}}=n^{\prime}, m^{\prime} \in \mathcal{M}_{m}\right\}\right| \\
& -a\left|\left\{m^{\prime}: s_{m^{\prime}}=n, m^{\prime} \in \mathcal{M}_{m}\right\}\right| .
\end{aligned}
$$

The corresponding change in total cost is,

$$
\begin{aligned}
& \Delta C\left(\stackrel{\curvearrowright}{n n^{\prime}}, \mathbf{s}_{-m}\right)=a\left|\left\{m^{\prime}: s_{m^{\prime}}=n^{\prime}, m^{\prime} \in \mathcal{M}_{m}\right\}\right| \\
& -a\left|\left\{m^{\prime}: s_{m^{\prime}}=n, m^{\prime} \in \mathcal{M}_{m}\right\}\right| \\
& +\Delta c\left(\stackrel{\curvearrowright}{n n^{\prime}, \mathbf{s}_{-m}}\right) \text {, } \\
& =2 \Delta c\left(\stackrel{\curvearrowright}{n n^{\prime}}, \mathbf{s}_{-m}\right) \text {. }
\end{aligned}
$$

From (2) and (3), the total system cost $C(\mathbf{s})$, is a weighted potential function of $\mathcal{G}_{\mathcal{M}}$. The weight in this case is 2 .

Now that the existence of NE is established, the next important step in the discussion is to compare the performance of NE solutions against the performance of the optimal solution. The optimal solution solves the problem

$$
\underset{\mathbf{s} \in \mathcal{N}^{M}}{\operatorname{minimize}} C(\mathbf{s})
$$

\section{EFFICIENCY OF EQUILIBRIA}

In general two parameters define the efficiency of $\mathrm{NE}$ of a game: price of stability and price of anarchy. The price of stability of a game is the ratio of the minimum cost achievable over all NEs and the optimal cost. The price of anarchy is the ratio of the worst cost over all NEs and the optimal cost [19].

The following lemma establishes that there are NEs of the game $\mathcal{G}_{\mathcal{M}}$ which are as good as the optimal solution of (4).

Lemma 2. The price of stability of $\mathcal{G}_{\mathcal{M}}$ is 1 .

Proof: The proof is by contradiction. Suppose $\mathbf{s}^{*}$ is an optimal assignment which minimizes the social cost $C(\cdot)$. Then let the BSs perform BR dynamics starting from $\mathbf{s}^{*}$. If a BR exist of at least one $m \in \mathcal{M}$ then the social cost $C(\cdot)$ decreases and $\mathbf{s}^{*}$ is not optimal. Therefore there must not exist a BR for any player at the optimal assignment $\mathbf{s}^{*}$. Hence by the definition of NE the optimal assignment is a pure strategy $\mathrm{NE}$ and the price of stability of $\mathcal{G}_{\mathcal{M}}$ is 1 .

Lemma 2 is optimistic. Anyhow, a bound for price of anarchy is required to be certain that the the worst case NE performance may not be far from the optimal performance. In the following discussion, it is helpful to imagine the assignment as a routing game on a directed graph $G_{R}$ of two nodes $\{S, T\}$ and $N$ parallel edges from $S$ to $T$. Then, in routing terms, all BSs which share the same PCI are on the same edge. But this does not mean that the price of anarchy of an atomic routing game applies to $\mathcal{G}_{\mathcal{M}}$. The fundamental difference between a routing game and the PCI assignment is that in a routing game the cost of taking route $n \in \mathcal{N}$ for player $m \in \mathcal{M}$, is a function of all players who take route $n$. Whereas in PCI assignment the cost is $c_{m}$, is a function of players who take route $n$ and who belong to $\mathcal{M}_{m}$. Due to this difference in cost function, the bounds given in [19], for routing games with linear cost functions are not applicable to game $\mathcal{G}_{\mathcal{M}}$. Fig. 2 provides an example routing graph $G_{R}$ where $m^{\prime} \in \mathcal{M}_{m}$ but $m^{\prime \prime} \notin \mathcal{M}_{m}$.

Lemma 3. The price of anarchy of $\mathcal{G}_{\mathcal{M}}$ is upper bounded by $\frac{\sum_{m} a \cdot M_{m}}{d \cdot M}+1, d>0$.

Proof: The proof is discussed with reference to Fig. 2. Suppose $\mathbf{s}^{*}$ is an optimal assignment which solves (4) and $\breve{\mathbf{s}}$ is a NE assignment of $\mathcal{G}_{\mathcal{M}}$. For a given assignment $\mathbf{s}$ the number of neighbors of $m \in \mathcal{M}$ on edge $s_{m} \in \mathcal{N}$ of the routing graph $G_{R}$ of Fig. 2, is denoted by $x_{m n}(\mathbf{s})$.

$$
\begin{gathered}
a x_{m \breve{s}_{m}}(\breve{\mathbf{s}}) \leq a x_{m s_{m}^{*}}(\breve{\mathbf{s}}), \\
a x_{m \breve{s}_{m}}(\breve{\mathbf{s}}) \leq a x_{m s_{m}^{*}}(\breve{\mathbf{s}})+a x_{m s_{m}^{*}}\left(\mathbf{s}^{*}\right) .
\end{gathered}
$$

Summing over $m$,

$$
\begin{gathered}
C(\breve{\mathbf{s}}) \leq \sum_{m} a x_{m s_{m}^{*}}(\breve{\mathbf{s}})+C\left(\mathbf{s}^{*}\right), \\
\frac{C(\breve{\mathbf{s}})}{C\left(\mathbf{s}^{*}\right)} \leq \frac{\sum_{m} a x_{x_{m s_{m}^{*}}}(\breve{\mathbf{s}})}{C\left(\mathbf{s}^{*}\right)}+1 .
\end{gathered}
$$

Note that $\frac{\sum_{m} a x_{m s_{m}^{*}}(\breve{\mathbf{s}})}{C\left(\mathbf{s}^{*}\right)} \leq \frac{\sum_{m} a M_{m}}{M d}$. Therefore

$$
\frac{C(\breve{\mathbf{s}})}{C\left(\mathbf{s}^{*}\right)} \leq \frac{\sum_{m} a M_{m}}{d M}+1
$$




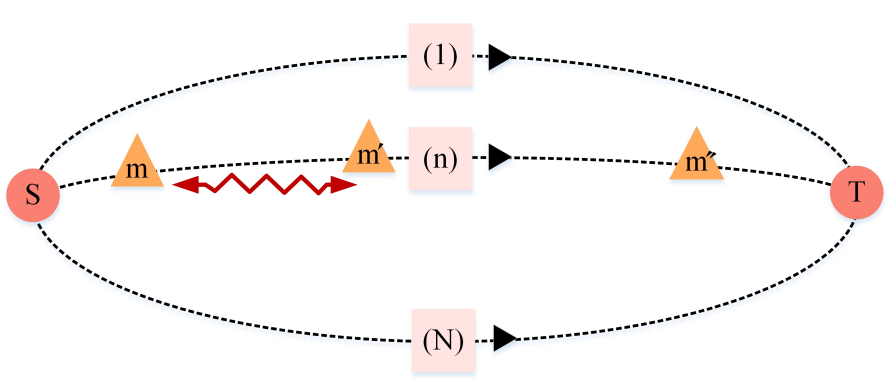

Figure 2: The routing graph $G_{R}$, of game $\mathcal{G}_{\mathcal{M}}$. The cost of BS $m$ on link $n$ depends only on the number of neighbors of $m$ that are on $n$.

The tightness of the bound (5) is yet to be verified. The price of anarchy is not defined for $C\left(\mathbf{s}^{*}\right)=0$, therefore price of anarchy for $d=0$ is not discussed. The following lemma refines the bound for the case when $N$ is larger than the maximum degree of a graph. The degree of a node $m \in \mathcal{M}$ is $M_{m}$ and maximum degree of graph $G$ is $\Delta(G):=\max _{m \in \mathcal{M}} M_{m}$.

Lemma 4. Given $N>\Delta(G)$ all $N E$ of $\mathcal{G}_{\mathcal{M}}$ are socially efficient with zero collisions.

Proof: The proof is by contradiction. Suppose there is a NE assignment $\mathbf{s}$, where a BS $m \in \mathcal{M}$ has collisions. By assumption the number of neighbors of $m$ is $M_{m}<N$. Therefore there exists at least one $n \in \mathcal{N}$, which is not taken by some $m^{\prime} \in \mathcal{M}_{m}$. Therefore $m$ is not playing a BR in the assignment $\mathbf{s}$ and therefore by definition of $\mathrm{NE}, \mathrm{s}$ is not a NE. Therefore at any NE assignment $\breve{s}$, all players have zero collisions. Therefore all NEs are globally optimal for (4) and therefore the price of anarchy is 1 .

\section{Simultaneous Synchronous Update Algorithm}

It is well known that the sequential BR dynamics converges to a NE in all potential games. On the other hand simultaneous synchronous BR dynamics generally do not converge to a NE [19]. Sequential dynamics are not highly alluring, as only a single player performs an action at a time and also a mechanism is needed to select the next player to perform the BR.

This section proposes a randomized simultaneous synchronous update algorithm which converges to the NE of game $\mathcal{G}_{\mathcal{M}}$, for $N>\Delta(G)$. Note that the game $\mathcal{G}_{\mathcal{M}}$ does not consider any restriction on the cardinality of resource set $N$. This algorithm assumes that the nodes posses the knowledge of $\Delta(G)$. The algorithm assumes message level synchronization, as opposed to symbol level. Lemma 4 dictates that for $N>\Delta(G)$, all NEs are optimal and have zero collisions. Conversely any zero collision assignment is a NE. Therefore the objective of the algorithm is to assign the set $\mathcal{N}$, to BSs, such that neighbors receive different PCIs and therefore all BSs achieve zero collisions. The proposed algorithm is given in Algorithm 1. The algorithm is local to each BS, i.e., each BS runs an instance of Algorithm 1. The notation, $c_{m}(t)$ denotes the number of collisions of the BS $m \in \mathcal{M}$ at time $t$ and $C(t)=\sum_{m \in \mathcal{M}} c_{m}(t)$ denotes sum collisions at time $t \in \mathbb{N}_{0}$. The random variable in PCI set $\mathcal{N}$, of player $m \in \mathcal{M}$, at time $t$, is denoted by $p_{m}(t, n)$. The sampled PCI at time $t$ by player $m$ is denoted by $s_{m}(t)$.

Initially at time $t=0$, BS $m$, equiprobably samples a PCI from $\mathcal{N}$, call this $s_{m}(0)$. Then BS $m$ announces its choice to its neighbors $m^{\prime} \in \mathcal{M}_{m}$, by the message $\left[s_{m}(0)\right]$ and simultaneously listens to the messages from neighbors. A neighbor message can be of two types. It may be a PCI $\left[s_{m^{\prime}}(t)\right]$, or it may be a single bit message set to [1]. The messages $\left[s_{m^{\prime}}(t)\right]$ are from $m^{\prime} \in \mathcal{M}_{m}$ who have still not acquired a PCI and the single bit message [1] are from $m^{\prime} \in \mathcal{M}_{m}$ who have already acquired a PCI, which is same as the one announced by $m$, i.e. $s_{m}(0)=s_{m \prime}(0)$. By comparing with neighbor messages BS $m$ can calculate the number of collisions $c_{m}(0)$, at $t=0$ and verify if the initial pick $s_{m}(0)$ collides with PCI choices of its neighbors. If it is collision free, i.e., $c_{m}(0)=0$, BS $m$ acquires $s_{m}(0)$, for the rest of its life time and $m$ is said to be in acquired state. If the initial PCI, $s_{m}(0)$, of $m \in \mathcal{M}$ experienced collisions, i.e., $c_{m}(0)>0$, then $m$ resamples uniformly from $\mathcal{N}$ and again shares the new PCI information $s_{m}(t)$ with the neighbors by sending the message $\left[s_{m}(t)\right]$ and simultaneously listens to neighbor messages to verify collisions for $s_{m}(t)$ by calculating $c_{m}(t)$. BS $m$ repeats this process till there is a time $t_{m}$ where all received messages from the neighbors suggest that there is no collision with $s_{m}\left(t_{m}\right)$ and then BS $m$ goes to acquired state with $s_{m}\left(t_{m}\right)$. Once in acquired state $m$ continues to listen and sends single bit message [1] to the unacquired set of neighbors $\check{\mathcal{M}}_{m} \subset \mathcal{M}_{m}$ who are in collision with $s_{m}\left(t_{m}\right)$.

The key difference in Algorithm 1 from [15], [16] is that at each iteration, full set $\mathcal{N}$ is sampled, without conditioning on neighbors. An added advantage is that, a node need not maintain a list of choices of its neighbors. The following lemma proves finite time convergence. Notation $\mathrm{P}(\cdot)$ denotes probability.

Lemma 5. For $N>\Delta(G)$, Algorithm 1 converges to a 0 collision state in finite time with probability 1.

Proof: The proof is based on first Borel-Cantelli lemma. The proof must show that $\sum_{t \in \mathbb{N}_{0}} \mathrm{P}(C(t)>0)<\infty$. Without loss of generality, let us consider a BS $m \in \mathcal{M}$, which has not acquired a PCI yet. Suppose that by time $t \geq 0, k \leq M_{m}$ out of the $M_{m}$ neighbors of $m$ have acquired a PCI. Then the probability that $m$ acquires a PCI in the next time slot is given by $\frac{N-k}{N}\left(1-\frac{1}{N}\right)^{M_{m}-k}$. Note that

$$
\begin{aligned}
\frac{N-k}{N}\left(1-\frac{1}{N}\right)^{M_{m}-k} & \geq \frac{N-\Delta(G)}{N}\left(1-\frac{1}{N}\right)^{M_{m}} \\
& \geq \frac{1}{\Delta(G)+1}\left(1-\frac{1}{\Delta(G)+1}\right)^{M_{m}} \\
& \geq \frac{1}{\Delta(G)+1}\left(1-\frac{1}{\Delta(G)+1}\right)^{\Delta(G)+1} \\
& \geq \frac{1}{\Delta(G)+1} \cdot \frac{1}{4} \geq \frac{1}{8 \Delta(G)}
\end{aligned}
$$

Let $U(t)$ denote the number of unacquired nodes at the beginning of round $t$. Then by the above argument, in expectation, at least $\frac{1}{8 \Delta(G)} U(t)$ number of nodes acquire a PCI in the next 


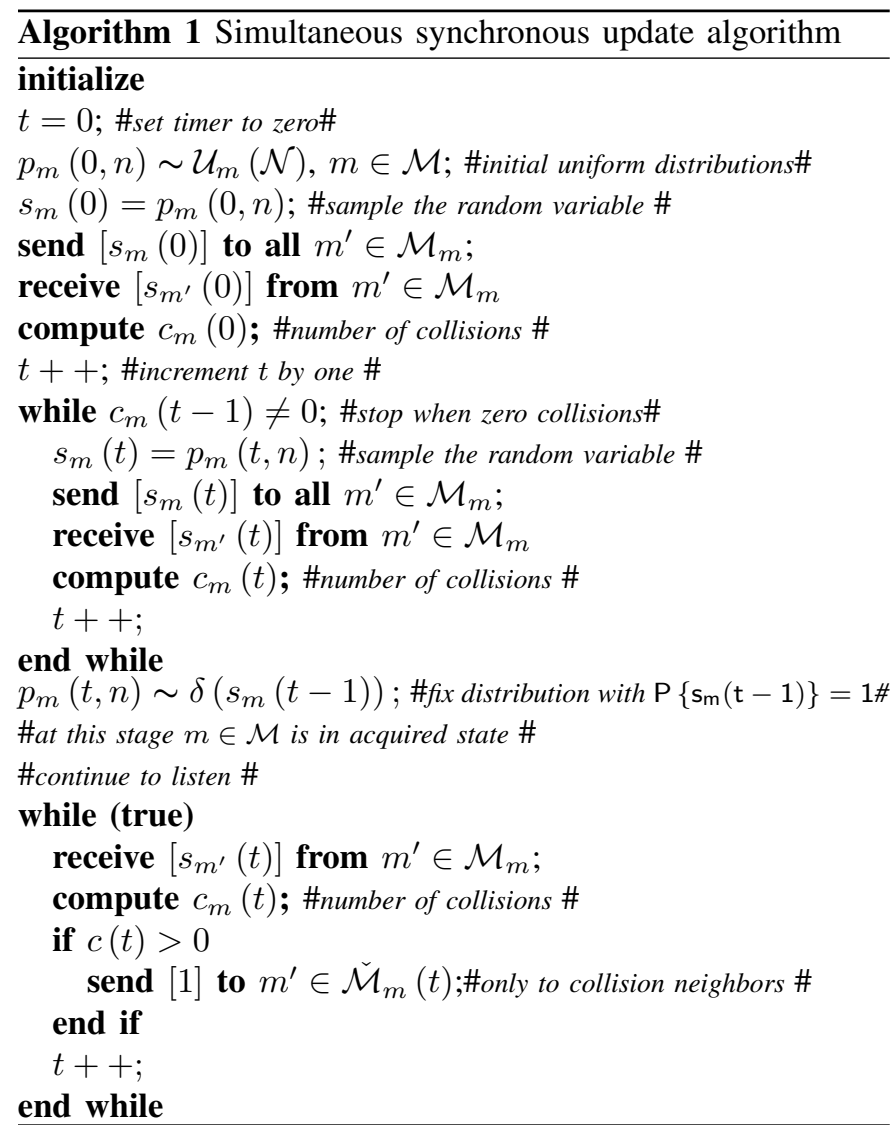

trial period. By backward recursion till $t=0$,

$\mathrm{E}(U(t)) \leq \mathrm{E}(U(0))\left(1-\frac{1}{8 \Delta(G)}\right)^{t}=M\left(1-\frac{1}{8 \Delta(G)}\right)$

Then by Markov's inequality $\mathrm{P}(U(t) \geq 1) \leq \mathrm{E}(U(t)) \leq$ $M\left(1-\frac{1}{8 \Delta(G)}\right)^{t}$. For there to be a collision at time $t>0$, there needs be at least one unacquired node at time $t-1 \geq 0$. Therefore $\mathrm{P}(C(t)>0) \leq \mathrm{P}(U(t-1)>1)$ and then

$$
\begin{aligned}
\sum_{t \in \mathbb{N}_{0}} \mathrm{P}(C(t)>0) & \leq 1+\sum_{t \in \mathbb{N}_{>0}} \mathrm{P}(U(t-1) \geq 1) \\
& \leq 1+\sum_{t \in \mathbb{N}_{>0}} M\left(1-\frac{1}{8 \Delta(G)}\right)^{t-1} \\
& \leq 1+8 M \Delta(G)
\end{aligned}
$$

Finally by the first Borel-Cantelli lemma, since $\sum_{t \in \mathbb{N}_{0}} \mathrm{P}(C(t)>0)<\infty$, collisions cease after a finite time with probability 1 [20]. By Lemma 4, ceasing of collisions is equivalent to each BS acquiring a PCI. Additionally, $\mathrm{P}(U(t)=0) \geq 1-M\left(1-\frac{1}{8 \Delta(G)}\right)^{t}>1-M \exp \left(\frac{-t}{8 \Delta(G)}\right)$. Letting $t=8 \delta \Delta(G) \log M$ for some constant $\delta>0$, $\mathrm{P}(U(t)=0)>1-\frac{1}{M^{\delta}}$. Thus, in $O(\delta \Delta(G) \log M)$ rounds, Algorithm 1 converges with probability $1-\frac{1}{M^{\delta}}$.

Remark 1. (Robustness of Algorithm 1) Removal of an edge or a node does not affect Lemma 5. Consider addition of an edge to graph $G$, to form $G^{\prime}$ still $N>\Delta\left(G^{\prime}\right)$. Adding a new edge to node $m \in \mathcal{M}$, does not affect it if $m$ is already in acquired state. If $m$ has still not acquired, then Lemma 5 holds for $G^{\prime}$ and $m$ eventually acquires. Therefore Algorithm 1 is robust to addition or removal of BSs and neighbor relations (edges). To demonstrate non-robustness of $N<\Delta(G)+1$, consider a star graph, where center node has $K$ neighbors, $\Delta(G)=K$. If initially center node is missing and Algorithm 1 has $N<\Delta(G)+1$, there is a non-zero probability that all colors are used by the outer nodes. When the center node comes on, some of the nodes have to be reassigned.

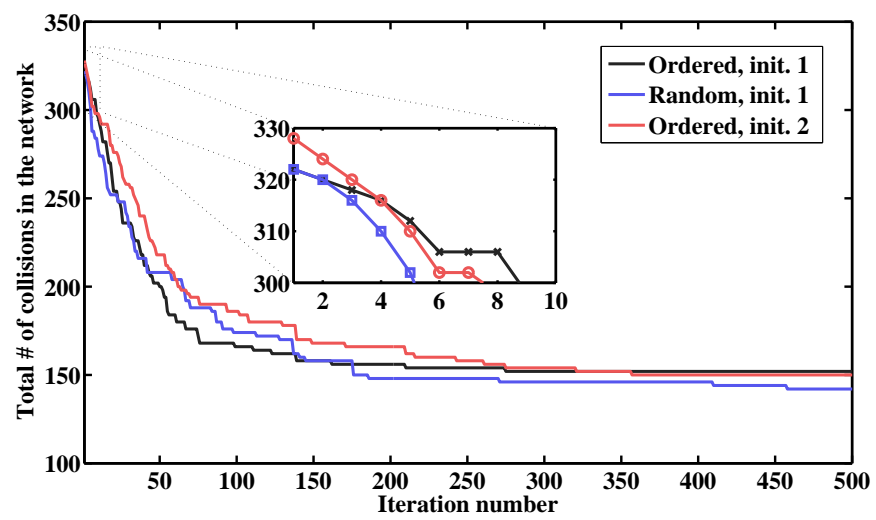

Figure 3: Convergence of BR dynamics and sub-optimality of Nash equilibria.

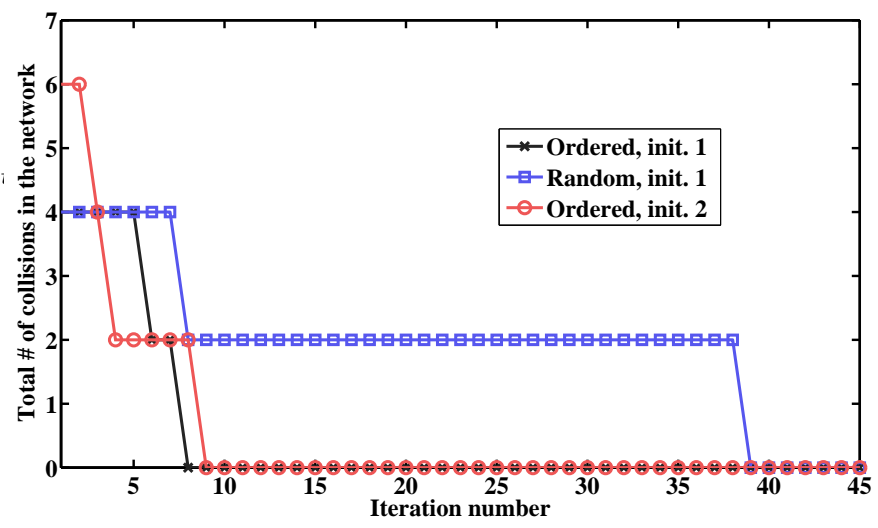

Figure 4: Numerical analysis of Lemma 4, with $\max _{m \in \mathcal{M}} M_{m}=9$ and 10 number of PCIs.

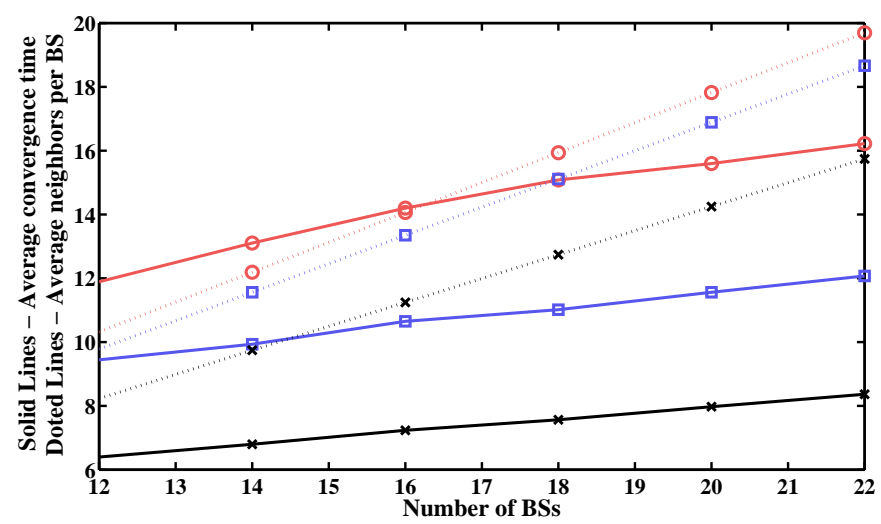

Figure 5: The convergence time of Algorithm 1 is sub-linear in the size of the graph $G$. 


\section{Numerical RESUlts}

Fig. 3 demonstrates the convergence of BR dynamics to NEs of $\mathcal{G}_{\mathcal{M}}$, hence it verifies that $C(\mathbf{s})$ is a potential function. The simulation environment for Fig. 3 assumes 60 BSs and 5 PCIs. To emphasize on the number of collisions, the cost function parameters are set to, $a=1, d=0$. Therefore the value of sum of cost functions reads the number of collisions. The adjacency matrix, which defines the edges of the graph $G=$ $(\mathcal{M}, \mathcal{E})$, is randomly generated and the maximum number of neighbors for any BS, i.e., $\Delta(G)$, is 41 and the minimum is 18 with an average of 30 neighbors per BS. Two different initial random assignments are considered, init. 1 and init. 2. Also two mechanisms are used to pick the next BS to perform BR dynamics. The first is named "ordered", where BSs are picked according to their index and at the end of the queue circles back to the first BS. The second mechanism is named "random" picks BSs randomly. As can be observed from Fig. 3 , the NE depends on both, the initial assignment and the mechanism for picking the next BS. All three curves converge to a their respective NEs.

To investigate Lemma 4 the network is setup with 14 BSs. The $\Delta(G)=9$ and the number of PCIs is 10 . The results of BR dynamics are shown in Fig. 4. It is clear that all NEs are achieved with zero collisions and therefore are optimal irrespective of the initial assignment or the BR dynamics, BS selection mechanism.

Fig. 5 shows the convergence time of Algorithm 1. The number of PCIs is set to $\Delta(G)+1$. Sequential BR for the case $N>\Delta(G)$ requires exactly $M$ iterations to terminate, since each BS needs a chance to verify (and change) its initial assignment. Therefore BR dynamics are linear in the size of the number of BSs. On the other hand, Fig. 5 demonstrates that the empirical average of number of iterations for Algorithm 1 is sub-linear in both the number of BSs $M$, and the average number of neighbors per BS, i.e., average degree of a node in $G$. That is an extremely powerful observation because Algorithm 1 outperforms BR dynamics.

\section{CONCLUSION}

This paper considered the problem of assigning a finite, $N$ number of resource, to minimize collisions among neighboring cells in a wireless network of $M$ cells. It is shown that the minimum-collisions resource assignment is NP-complete by a reduction of graph coloring problem. The analysis is discussed in the context of physical cell identifier assignment problem. The paper proposed a non-cooperative potentialgame-theoretic solution and demonstrated that the price of stability is 1 . Moreover the price of anarchy is upper bounded. In addition a novel fully distributed randomized algorithm for the special case, where the number of neighbors of any node of the network is less than $N$ is developed. The algorithm is proven to converge to a Nash equilibrium solution in finite time and the robustness of the algorithm to addition or deletion of cells and neighbor relations is also proved. Simulations demonstrated that the algorithm grows sub-linearly in the size of the network and therefore outperforms best response dynamics. Several paths remain to be further explored. The tightness of the upper bound for price of anarchy must be investigated. Sub-linearity of the algorithm can be analytically explored. The possibility of a randomized algorithm for the case where the number of neighbors of a BS is higher than $N$ can also be a future extension of this paper.

\section{Acknowledgment}

We are thankful to Dr. Yuval Filmus of Institute for Advanced Study (IAS), Princeton for his comments on NPcompleteness of the problem.

\section{REFERENCES}

[1] M. Peng, D. Liang, Y. Wei, J. Li, and H.-H. Chen, "Selfconfiguration and self-optimization in LTE-advanced heterogeneous networks," vol. 51, no. 5, pp. 36-45, May 2013.

[2] 3GPP, "LTE; evolved universal terrestrial radio access (E-UTRA); physical channels and modulation (3GPP TS 36.211 version 11.5.0 Release 11)," ETSI, Tech. Rep., 2013.

[3] P. N. Panagopoulou and P. G. Spirakis, "A game theoretic approach for efficient graph coloring," in Proc. 19th International Symposium on Algorithms and Computation, (ISAAC '08), 2008, pp. 183-195.

[4] B. Escoffier, L. Gourves, and J. Monnot, "Strategic coloring of a graph," Internet Mathematics, vol. 8, no. 4, pp. 424-455, 2012.

[5] T. Moscibroda and R. Wattenhofer, "Coloring unstructured radio networks," Distributed Computing, vol. 21, no. 4, pp. 271-284, 2008.

[6] B. Derbel and E. Talbi, "Distributed node coloring in the sinr model," in Proc. IEEE 30th International Conference on Distributed Computing Systems, (ICDCS '10), Jun. 2010, pp. 708-717.

[7] M. Halldorsson, J. Halpern, E. Li, and V. Mirrokni, "On spectrum sharing games," Distributed Computing, vol. 22, no. 4, pp. 235-248, 2010.

[8] N. Nie and C. Comaniciu, "Adaptive channel allocation spectrum etiquette for cognitive radio networks," Springer-Verlag Mob. Netw. Appl., vol. 11, no. 6, pp. 779-797, Dec. 2006.

[9] Y. Liu, L. Dong, and R. Marks, "Common control channel assignment in cognitive radio networks using potential game theory," in Proc. IEEE Wireless Communications and Networking Conference, (WCNC '13), Apr. 2013, pp. 315-320.

[10] J. Oppolzer and R. Bestak, "Cell identifier assignment in two-tier femtocell networks," in Proc. IEEE Vehicular Technology Conference, (VTC Spring '13), Jun. 2013, pp. 1-5.

[11] M. Amirijoo, P. Frenger, F. Gunnarsson, H. Kallin, J. Moe, and K. Zetterberg, "Neighbor cell relation list and physical cell identity self-organization in LTE," in Proc. IEEE International Conference on Communications, (ICC '08), May 2008, pp. 37-41.

[12] X. Zhang, E. Liu, Z. Xiao, and J. Zhang, "Dynamic PCI assignment in two-tier networks based on cell activity prediction," IEEE/IET Electron. Lett., vol. 49, no. 24, pp. 1570-1572, Nov. 2013.

[13] T. Bandh, G. Carle, and H. Sanneck, "Graph coloring based physicalcell-id assignment for lte networks," in Proc. International Conference on Wireless Communications and Mobile Computing: Connecting the World Wirelessly, (IWCMC '09), 2009, pp. 116-120.

[14] Y. WEI, W. bo WANG, M. gen PENG, and S. jun MIN, "Graph theory based physical cell identity self-configuration forLTE-A network," The Journal of China Universities of Posts and Telecommunications, vol. 20, pp. $101-107,2013$

[15] K. Chaudhuri, G. Chung, and M. Jamall, "A network coloring game," in Proc. Internet and Network Economics, ser. Lecture Notes in Computer Science. Springer Berlin Heidelberg, 2008, vol. 5385, pp. 522-530.

[16] T. Bohme and J. Schreyer, "Local computation of vertex colorings," in Proc. Cologne-Twente Workshop on Graphs and Combinatorial Optimization, (CTW'11), Jun. 2011, pp. 76-78.

[17] J. Schneider and R. Wattenhofer, "Coloring unstructured wireless multihop networks," in Proc. 28th ACM Symposium on Principles of Distributed Computing, (PODC '09), Aug. 2009, pp. 210-219.

[18] F. Ahmed, O. Tirkkonen, M. Peltomäki, J. Koljonen, C. Yu, and M. Alava, "Distributed graph coloring for self-organization in LTE networks," JECE, vol. 2010, pp. 5:1-5:10, Jan. 2010.

[19] N. Nisan, T. Roughgarden, E. Tardos, and V. V. Vazirani, Algorithmic game theory. Cambridge University Press, 2007.

[20] D. Williams, Probability with Martingales, ser. Cambridge mathematical textbooks. Cambridge University Press, 1991. 